\title{
Hybrid Difference Methods for the Initial Boundary-Value Problem for Hyperbolic Equations
}

\author{
By Joseph Oliger*
}

\begin{abstract}
The use of lower order approximations in the neighborhood of boundaries coupled with higher order interior approximations is examined for the mixed initial boundary-value problem for hyperbolic partial differential equations. Uniform error can be maintained using smaller grid intervals with the lower order approximations near the boundaries. Stability results are presented for approximations to the initial boundary-value problem for the model equation $u_{t}+c u_{x}=0$ which are fourth order in space and second order in time in the interior and second order in both space and time near the boundaries. These results are generalized to a class of methods of this type for hyperbolic systems. Computational results are presented and comparisons are made with other methods.
\end{abstract}

1. Introduction. It has been established that fourth order methods are much more efficient than those of first and second order for hyperbolic partial differential equations [5], [9], [11]. When such methods are used for the initial boundary-value problem, awkward situations arise in the neighborhood of the boundaries since the interior approximations cannot be used there in a straightforward manner. It is attractive to consider matching lower order approximations in the neighborhood of the boundaries to higher order interior approximations. However, it has been established by Gustafsson [6] that more than one order of accuracy cannot be dropped near the boundaries without sacrificing the rate of convergence over the entire region. Computational examples [6], [11] illustrate this fact. Consequently, a denser net must be used with the lower order approximation if the overall accuracy is to be maintained.

There are many applications where this approach is quite natural for other reasons. For example, oceanographic problems often have boundaries and associated boundary layer phenomena which are quite complex compared to the solution in the interior. A very fine grid may be necessary to adequately represent these boundaries and lower order approximations may be appropriate in the boundary layer since the boundary influence is often of a forced rather than a transient nature (see [5], [9] for details of the error as a function of time for approximations of different orders of accuracy).

In Section 2 we begin by examining methods for the model problem

$$
\begin{gathered}
u_{t}+c u_{x}=0, \quad c<0, a \leqslant x \leqslant b, t \geqslant 0 ; \\
u(x, 0)=f(x), \quad a \leqslant x \leqslant b
\end{gathered}
$$

Received November 18, 1975.

AMS (MOS) subject classifications (1970). Primary 65M10.

* This work has been supported in part by the National Science Foundation under grants DCR72-03712 A03 and GJ-29988X and by the Energy Research and Development Adminstration under contract $\mathrm{E}(04-3)$ 326-PA \# 30. 


$$
u(b, t)=g(t), \quad t \geqslant 0 ;
$$

with compatibility condition $f(b)=g(0)$. We first consider a centered difference approximation to (1.1)-(1.3) which is fourth order in space and second order in time in the interior coupled with the second order leapfrog method near the boundaries. This method is found to be unstable unless the same grid interval is used with both the leapfrog and more accurate interior approximation. Consequently, this method has limited usefulness. We also consider using the Lax-Wendroff approximation near the boundaries. This combined method is found to be stable. We conclude Section 2 with general results for methods of this type for hyperbolic systems.

In Section 3 we present numerical results obtained using the methods presented in Section 2 and compare these results with those obtained in [11] where uncentered approximations of third order were used in the neighborhood of the boundaries.

We will use the theory of Gustafsson, Kreiss and Sundström [7] and assume that the reader is familiar with the results of that paper. The stability results presented here for constant coefficients can be extended to the variable coefficient case in the same manner as those of [7].

2. The Methods and Stability Results. We begin by examining an approximation to (1.1), (1.2) and (1.3). We can take $a=0$ and $b=1$ without loss of generality. Let $k>0$, $h_{c}=1 / N$ and $h_{f}=h_{c} / M$ where $N$ and $M$ are natural numbers. Let $\lambda_{c}=k / h_{c}$ and $\lambda_{f}=$ $k / h_{f}$. Define grid functions $v_{\nu}(t)=v\left(\nu h_{c}, t\right)$ for $\nu=0,1, \ldots, N ; l_{\nu}(t)=l\left(\nu h_{f}, t\right)$ for $\nu=0,1, \ldots, 2 M$ and $r_{\nu}(t)=r\left(1-h_{c}+\nu h_{f}, t\right)$ for $\nu=0,1, \ldots, M$ where $t=$ $0, k, 2 k, \ldots$ (see Figure 1). For $2 \leqslant \nu \leqslant N-2$ we approximate (1.1) by the $O\left(h_{c}^{4}+k^{2}\right)$ approximation
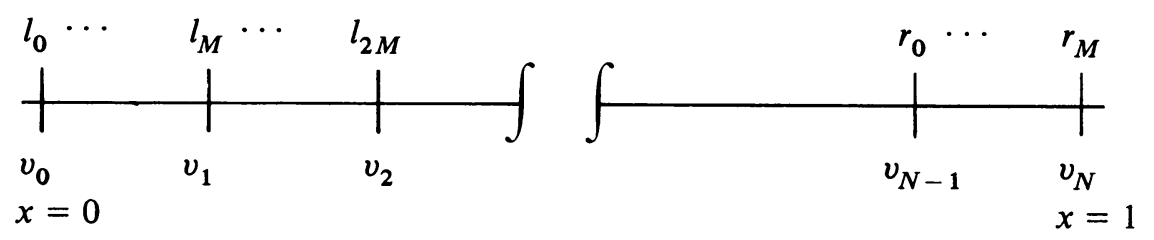

\section{Figure 1}

$$
v_{\nu}(t+k)=v_{\nu}(t-k)-c 2 k\left[\frac{4}{3} D_{0}\left(h_{c}\right)-\frac{1}{3} D_{0}\left(2 h_{c}\right)\right] v_{\nu}(t),
$$

where $D_{0}\left(n h_{c}\right) v_{\nu}(t)=\left(2 n h_{c}\right)^{-1}\left[v_{\nu+n}(t)-v_{\nu-n}(t)\right]$. On the interval $\left[0,2 h_{c}\right]$ we approximate $(1.1)$ by the $O\left(h_{f}^{2}+k^{2}\right)$ approximation

$$
l_{\nu}(t+k)=l_{\nu}(t-k)-c 2 k D_{0}\left(h_{f}\right) l_{\nu}(t) \text { for } \nu=1,2, \ldots, 2 M-1,
$$

and at $x=0$ by the $O\left(h_{f}+k^{2}\right)$ approximation

$$
l_{0}(t+k)=l_{0}(t-k)-c 2 \lambda_{f}\left[l_{1}(t)-0.5\left(l_{0}(t-k)+l_{0}(t+k)\right)\right] .
$$

On the interval $\left[1-h_{c}, 1\right]$ we approximate $(1.1)$ by the similar $O\left(h_{f}^{2}+k^{2}\right)$ and $O\left(h_{f}+k^{2}\right)$ formulae

$$
r_{\nu}(t+k)=r_{\nu}(t-k)-c 2 k D_{0}\left(h_{f}\right) r_{\nu}(t) \text { for } \nu=1,2, \ldots, M-1
$$


and

$$
r_{0}(t+k)=r_{0}(t-k)-c 2 \lambda_{f}\left[r_{1}(t)-0.5\left(r_{0}(t+k)+r_{0}(t-k)\right)\right] .
$$

Corresponding to the initial condition (1.2), we use

$$
\begin{array}{ll}
v_{\nu}(0)=f\left(\nu h_{c}\right) & \text { for } \nu=0,1, \ldots, N \\
l_{\nu}(0)=f\left(\nu h_{f}\right) & \text { for } \nu=0,1, \ldots, 2 M
\end{array}
$$

and

$$
r_{\nu}(0)=f\left(1-h_{c}+\nu h_{f}\right) \text { for } \nu=0,1, \ldots, M .
$$

Corresponding to the boundary condition (1.3), we use

$$
r_{M}(t)=g(t) \text { for } t=0, k, 2 k, \ldots
$$

We then link the grid functions $l_{\nu}(t), v_{\nu}(t)$ and $r_{\nu}(t)$ by

$$
\begin{gathered}
v_{0}(t)=l_{0}(t), \quad v_{1}(t)=l_{M}(t), \quad v_{2}(t)=l_{2 M}(t), \\
v_{N-1}(t)=r_{0}(t) \quad \text { and } \quad v_{N}(t)=r_{M}(t)
\end{gathered}
$$

for $t=0, k, 2 k, \ldots$.

We complete the specification by giving

$$
\begin{cases}v_{\nu}(k)=w\left(\nu h_{c}\right), & v=0,1, \ldots, N, \\ l_{\nu}(k)=w\left(\nu h_{f}\right), & v=0,1, \ldots, 2 M, \\ r_{\nu}(k)=w\left(1-h_{c}+\nu h_{f}\right), & v=0,1, \ldots, M,\end{cases}
$$

where $w$ is a sufficiently accurate approximation to the solution $u(x, t)$ at $t=k$.

It is clear that Eqs. (2.1), (2.2) and (2.3) determine a unique approximation which is consistent with the problem (1.1), (1.2) and (1.3).

The one-sided formulae (2.2b) and (2.3b) are due to A. Sundström, and it has been shown in Elvius and Sundström [4] that they yield stable approximations for the related initial boundary-value problems when used with the formulae (2.2a) and (2.3a). It is well known that (2.1a) is a stable approximation for the related Cauchy problem [5], [9].

Note that the approximations (2.2b) and (2.3b) are only $O\left(h_{f}+k^{2}\right)$ accurate. However, it follows from the results of Gustafsson [6] that overall convergence behavior is not adversely affected.

Assumption. We assume that $\lambda_{c}$ and $\lambda_{f}$ satisfy stability criteria which guarantee that our interior approximations are stable for the related Cauchy problems.

(2.1a), (2.2a) and (2.3a) are stable for the related Cauchy problems if $|c| \lambda_{f} \leqslant 1$ and $|c| \lambda_{c} \leqslant 6 / \sqrt{9+24 \sqrt{6}}=0.7287 \ldots$

We now investigate the stability of the method defined by (2.1), (2.2) and (2.3). We use the stability Definition 3.3 of Gustafsson et al. [7]. In [7], it is established (Theorem 5.4) that the stability of two related quarter-plane problems is equivalent to stability for the two-boundary problem in the sense of Definition 3.3. These two problems are simply obtained by removing one or the other of the boundaries and 
extending the domain to $\pm \infty$, as is appropriate. We will refer to these as the right and left quarter-plane problems.

It is immediate that the associated left quarter-plane problem, $-\infty<x \leqslant 1$, $t \geqslant 0$ (we extend $\nu$ over the negative integers in (2.1a)) is stable by Definition 3.3 of [7]. This follows from the fact that (2.1a) is stable for the related Cauchy problem and and that (2.3a) and (2.3b) are stable on the interval $\left[1-h_{c}, 1\right]$ and provide a $v_{N-1}(t)$ which is bounded on every finite $t$-interval in terms of the data $g(t)$. It is the independence of the calculation of the $r_{\nu}$ from the $v_{\nu}$ that makes this trivial.

The situation is more complicated for the associated right quarter-plane problem, $0 \leqslant x<\infty, t \geqslant 0$. First we must examine the stability of the approximation for the Cauchy problem given by (2.1a) with $\nu$ extended over all natural numbers and (2.2a) with $v$ extended over the negative integers. This is the problem of matching schemes investigated by Ciment [3] for dissipative approximations. This can also be analyzed in terms of the theory of [7], since we can think of folding the $x$-axis at zero and investigating the initial boundary-value problem for a vector $\left(v_{\nu}, \widetilde{l}_{M \nu}\right)^{\prime}$.

The new net structure is shown in Figure 2.

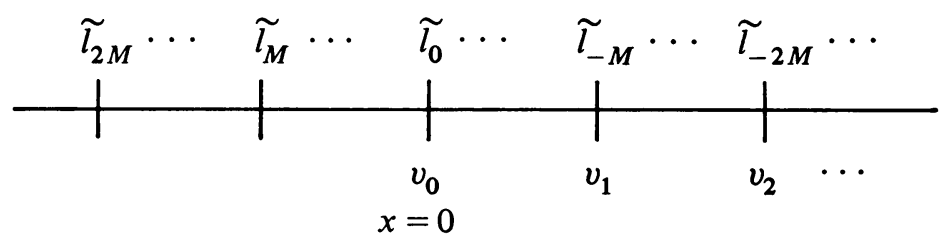

FIGURE 2

$\left(v_{\nu}, \tilde{l}_{M \nu}\right)^{\prime}$ is an approximation to the solution of the differential equation

$$
\left(\begin{array}{l}
u \\
w
\end{array}\right)_{t}=\left(\begin{array}{cc}
-c & 0 \\
0 & c
\end{array}\right)\left(\begin{array}{l}
u \\
w
\end{array}\right)_{x}, \quad 0 \leqslant x<\infty, t \geqslant 0,
$$

with boundary condition $w(0, t)=u(0, t)$. This technique has been used in [1], [2] and [3] where more detailed descriptions of this process can be found. Under this transformation the conditions $v_{2}(t)=l_{2 M}(t), v_{1}(t)=l_{M}(t)$ and $v_{0}(t)=l_{0}(t)$ become

$$
v_{2}(t)=\tilde{l}_{2 M}(t), \quad v_{1}(t)=\tilde{l}_{M}(t), \quad v_{0}(t)=\tilde{l}_{0}(t)
$$

and (2.2a) becomes

$$
\tilde{l}_{\nu}(t+k)=\tilde{l}_{\nu}(t-k)+c 2 k D_{0}\left(h_{f}\right) \tilde{l}_{\nu}(t)
$$

$$
\nu=2 M-1,2 M-2, \ldots, 0,-1,-2, \ldots .
$$

It is shown in [7] that stability according to their Definition 3.3 is equivalent to the fact that a determinantal equation (Eq. (10.3) of [7]) not vanish for complex $z$ such that $|z| \geqslant 1$. This determinantal equation can be derived formally by seeking the general solutions of (2.1a) and (2.2a') of the form $v_{\nu}(t)=\kappa^{\nu} z^{t / k}$ and $\tilde{l}_{\nu}(t)=\zeta^{\nu} z^{t / k}$ which belong to $l_{2}\left(h_{c}\right)$ and $l_{2}\left(h_{f}\right)$ for $t \geqslant 0$ and all complex $z$ such that $|z|>1$, i.e.,

$$
\left\|v_{\nu}(t)\right\|_{l_{2}\left(h_{c}\right)}^{2}=h_{c} \sum_{\nu=0}^{\infty}\left|v_{\nu}\right|^{2}<\infty
$$

and 


$$
\left\|\tilde{l}_{\nu}(t)\right\|_{l_{2}\left(h_{f}\right)}^{2}=h_{f} \sum_{\nu=2 M}^{-\infty}\left|\tilde{l}_{\nu}(t)\right|^{2}<\infty
$$

When this general solution is substituted into the boundary conditions $\left(2.1 c^{\prime}\right)$, a homogeneous system of linear equations for the arbitrary constants in the general solution is obtained. Let $C$ be the matrix of this system. The determinant condition (10.3) of [7] is $\operatorname{det} C \neq 0$ for $|z| \geqslant 1$. This is the requirement that there exist no nontrivial solutions of the assumed form for $|z| \geqslant 1$ which satisfy the boundary conditions. Our determinantal condition is equivalent to

$$
\operatorname{det}\left(\begin{array}{ccc}
1 & 1 & 1 \\
\kappa_{1} & \kappa_{2} & \zeta^{-M} \\
\kappa_{1}^{2} & \kappa_{2}^{2} & \zeta^{-2 M}
\end{array}\right) \neq 0 \quad \text { if } \kappa_{1} \neq \kappa_{2}
$$

and to

$$
\operatorname{det}\left(\begin{array}{ccc}
1 & 0 & 1 \\
\kappa_{1} & 1 & \zeta^{-M} \\
\kappa_{1}^{2} & 2 \kappa_{2} & \zeta^{-2 M}
\end{array}\right) \neq 0 \quad \text { if } \kappa_{1}=\kappa_{2} .
$$

$\kappa_{1}$ and $\kappa_{2}$ are the roots of the characteristic equation

$$
\kappa^{4}-8 \kappa^{3}-\frac{6\left(z^{2}-1\right)}{c \lambda z} \kappa^{2}+8 \kappa-1=0
$$

corresponding to $(2.1 \mathrm{a})$ such that $\left|\kappa_{i}\right| \leqslant 1 . \zeta$ is the root of the characteristic equation

$$
\zeta^{2}-\frac{\left(z^{2}-1\right)}{c \lambda z} \zeta-1=0
$$

corresponding to $\left(2.2 \mathrm{a}^{\prime}\right)$ such that $|\zeta| \leqslant 1$. The fact that $\kappa_{1}, \kappa_{2}$ and $\zeta$ are uniquely defined as the continuous functions of $z$ satisfying these criteria is established in [7]. It is also shown in [7] that $\left|\kappa_{i}\right|<1, i=1,2$ and $|\zeta|<1$ for $|z|>1$ so the conditions (2.4a) and (2.4b) are satisfied for $|z|>1$ since these determinants only vanish if $\kappa_{1}=$ $\zeta^{-M}$ or $\kappa_{2}=\zeta^{-M}$. In order to complete our analysis we must examine the roots $\kappa_{1}$, $\kappa_{2}$ of (2.5) and $\zeta$ of (2.6) for $z=e^{i \theta}$. To do this we need the following lemma.

LEMMA 2.1. Let $z=e^{i \theta}$ and $\kappa_{1}(\theta)$ and $\kappa_{2}(\theta)$ be the roots of $(2.5)$ which satisfy $\left|\kappa_{j}\right|<1, j=1,2$, when $|z|>1$. If we number properly, then $\left|\kappa_{1}\right|<1$ and $\left|\kappa_{2}\right| \leqslant 1$ for all $\theta$. Let $c<0$. Define $\theta_{1}$ to be the smallest positive value of $\theta$ such that

$$
\beta(\theta) \equiv \frac{12 \sin \theta}{c \lambda_{c}}=-\sqrt{36+96 \sqrt{6}}=-16.46 \ldots,
$$

then $0<\theta_{1} \leqslant \pi / 2$. Set $\theta_{2}=\pi-\theta_{1}$, then $\beta\left(\theta_{2}\right)=\beta\left(\theta_{1}\right)$. Define $\theta_{3}, \theta_{2}<\theta_{3}<\pi$, by $\beta\left(\theta_{3}\right)=-16$. Define $\theta_{4}, \pi<\theta_{4}<3 \pi / 2$, by $\beta\left(\theta_{4}\right)=16$. Let $\theta_{5}$ be the smallest value of $\theta$ such that $\beta(\theta)=\sqrt{36+96 \sqrt{6}}=16.46 \ldots$, then $3 \pi / 2<\theta_{5}<2 \pi$. Let $\theta_{6}=2 \pi-\theta_{5}$, then $\beta\left(\theta_{6}\right)=\beta\left(\theta_{5}\right)$. The $\theta_{j}$ so defined satisfy $0<\theta_{1} \leqslant \theta_{2}<\theta_{3}<\pi$ $<\theta_{4}<\theta_{5} \leqslant \theta_{6}<2 \pi . \theta_{1}=\theta_{2}$ and $\theta_{5}=\theta_{6}$ if and only if $\left|c \lambda_{c}\right|=12 / \sqrt{36+96 \sqrt{6}}$. The following properties of $\kappa_{2}(\theta)$ hold: 


$\begin{array}{rlrl}\kappa_{2} & =-1 & & \text { for } \theta=0 \\ \operatorname{Re} \kappa_{2} & <0,\left|\kappa_{2}\right|=1 & & \text { for } 0<\theta \leqslant \theta_{1} \\ \left|\kappa_{2}\right| & <1 & & \text { for } \theta_{1}<\theta<\theta_{2} \text { if } \theta_{1}<\theta_{2} \\ \operatorname{Re} \kappa_{2} & <0,\left|\kappa_{2}\right|=1 & & \text { for } \theta_{2} \leqslant \theta<\theta_{3} \\ \kappa_{2} & =i & & \text { for } \theta=\theta_{3} \\ \operatorname{Re} \kappa_{2} & >0,\left|\kappa_{2}\right|=1 & & \text { for } \theta_{3}<\theta<\pi \\ \kappa_{2} & =1 & & \text { for } \theta=\pi \\ \operatorname{Re} \kappa_{2} & >0,\left|\kappa_{2}\right|=1 & & \text { for } \pi<\theta<\theta_{4} \\ \kappa_{2} & =-i & & \text { for } \theta=\theta_{4} \\ \operatorname{Re} \kappa_{2}<0,\left|\kappa_{2}\right|=1 & & \text { for } \theta_{4}<\theta \leqslant \theta_{5} \\ \left|\kappa_{2}\right|<1 & & \text { for } \theta_{5}<\theta<\theta_{6} \text { if } \theta_{5}<\theta_{6} \\ \operatorname{Re} \kappa_{2}<0,\left|\kappa_{2}\right|=1 & & \text { for } \theta_{6} \leqslant \theta<2 \pi\end{array}$

If $c>0$, then $\kappa_{2}(0)=1$ and the above properties hold if we replace $\theta$ by $\theta^{\prime}=\theta-\pi$.

Proof. The properties of the $\theta_{j}$ follow easily from the assumption that (2.1a) is stable for the related Cauchy problem, i.e., $\left|c \lambda_{c}\right| \leqslant 12 / \sqrt{36+96 \sqrt{6}}$, and the properties of $\cos \theta$. It was shown in Lemma 2.1 of [11] that $\left|\kappa_{1}\right|<1$ and $\left|\kappa_{2}\right|<1$ if $\beta^{2}(\theta)>36+96 \sqrt{6}$ and that one of the $\kappa_{j}$ satisfies $\left|\kappa_{j}\right|=1$ and the other $\left|\kappa_{j}\right|<1$ for each value of $\theta$ such that $\beta^{2}(\theta) \leqslant 36+96 \sqrt{6}$. From our definition of the $\theta_{j}$, $\beta^{2}(\theta)>36+96 \sqrt{6}$ for $\theta_{1}<\theta<\theta_{2}$ if $\theta_{1} \neq \theta_{2}$ and for $\theta_{5}<\theta<\theta_{6}$ if $\theta_{5} \neq \theta_{6}$; and $\beta^{2}(\theta) \leqslant 36+96 \sqrt{6}$ otherwise. For $\beta \neq 0, \pm 8, \pm 16$ the number of roots of $(2.5)$ with positive real part, $p$, and the number with negative real part, $q$, are given by

$$
p=V\left(1,-8,64-\beta^{2}, 8 \beta^{2}, \beta^{2}\left(\beta^{2}-256\right)\right)
$$

and

$$
q=V\left(1,8,64-\beta^{2},-8 \beta^{2}, \beta^{2}\left(\beta^{2}-256\right)\right)
$$

where $V\left(a_{1}, \ldots, a_{n}\right)$ denotes the number of changes of sign in the real sequence $a_{1}, a_{2}, \ldots, a_{n}$ (Theorem (40.1) of [10]). We calculate:

$$
\begin{array}{ll}
p=3 \text { and } q=1 & \text { for } 0<|\beta|<8, \\
p=3 \text { and } q=1 & \text { for } 8<|\beta|<16, \\
p=2 \text { and } q=2 & \text { for } 16<|\beta| .
\end{array}
$$

Examining the roots of (2.5) at $z=1$ and at $z=1+\delta, \delta>0$, we find that $\kappa_{1}=$ $0.127 . \ldots, \kappa_{2}=-1$ and $p=3, q=1$ at $\theta=0$. By continuity, since $p=3, q=1$ for $0<|\beta|<8$ and since $\kappa= \pm i$ are roots of (2.5) if and only if $\beta=\mp 16$; we can conclude that $\kappa_{2}$ remains in the left half-plane for the $\theta$-neighborhood of 0 such that $0 \leqslant|\beta|<8$. Since $\pm i$ are not roots of $(2.5)$ for $\beta= \pm 8$ and $\left|\kappa_{2}\right|=1$, we can conclude that $\kappa_{2}$ remains in the left half-plane for the larger $\theta$-neighborhood of 0 such that $0 \leqslant$ $|\beta|<16$. Examination of the roots of (2.5) at $z=e^{i \theta_{1}}$ and $z=(1+\delta) e^{i \theta_{1}}$ shows $\kappa_{2}\left(\theta_{1}\right)=(-0.2247 \ldots)+i(0.9744 \ldots)$. Similarly, at $z=e^{i \theta_{6}}$ we find $\kappa_{2}\left(\theta_{6}\right)=$ $(-0.2247 .)+.i\left(-0.9744\right.$. . .). So, again by continuity, $\kappa_{2}(\theta)$ must remain in the left half-plane for $|\beta|>16$ since $p=2, q=2$ for all such $\beta$. Examination of the roots of (2.5) at $z=e^{i \theta_{3}}$ yields $\kappa_{2}\left(\theta_{3}\right)=i$, and at $z=e^{i \theta}$ we find $\kappa_{2}\left(\theta_{4}\right)=-i$ so 
it is $\kappa_{2}$ that moves into the right half-plane as we enlarge the $\theta$-neighborhood of 0 beyond $\theta_{3}$ and $\theta_{4}$. We can conclude that $\left|\kappa_{1}\right|<1$ and that $\operatorname{Re} \kappa_{2}<0$ for $0 \leqslant \theta<\theta_{3}$ and $\theta_{4}<\theta \leqslant 2 \pi$ and $\operatorname{Re} \kappa_{2}>0$ for $\theta_{3}<\theta<\theta_{4}$. This concludes the proof for $c<0$. The proof for $c>0$ proceeds similarly.

It follows from Lemma (6.2) of [7] and the formulae immediately preceding it that: (1) $|\zeta|=1$ and $\operatorname{sign}(\operatorname{Re} \zeta)=-\operatorname{sign}(c \operatorname{Re} z)$ when $z=e^{i \theta}$ and $\theta$ satisfies $|\sin \theta|<$ $\left|c \lambda_{f}\right|,(2)|\zeta|<1$ when $|\sin \theta|>\left|\lambda_{f} c\right|$, and (3) $\zeta=-1$ when $z=-1$ and $\zeta=1$ when $z=1$.

We now return to the examination of the determinant condition. We saw that it was satisfied for $|z|>1$ and now consider $z=e^{i \theta}$. It follows easily from Lemma 2.1 and the preceding paragraph that $\kappa_{1} \neq \zeta^{-M}$ since $\left|\kappa_{1}\right|<1$ and $\left|\zeta^{-M}\right| \geqslant 1$. Now we only have the condition $\kappa_{2} \neq \xi^{-M}$ remaining to examine. We consider three cases.

Case I, $M=1$. If $M=1$, then $\lambda_{c}=\lambda_{f}$ and

$$
\left|\frac{\sin \theta}{c \lambda_{f}}\right| \leqslant 1 \text { if and only if }|\beta(\theta)|=12\left|\frac{\sin \theta}{c \lambda_{f}}\right| \leqslant 12 \text {. }
$$

If $\left|\sin \theta / c \lambda_{f}\right| \leqslant 1$, then Lemma 2.1 implies that $\operatorname{sign}\left(\operatorname{Re} \kappa_{2}\right)=-\operatorname{sign}\left(\operatorname{Re} \zeta^{-1}\right)$ since $\operatorname{Re} \zeta=\operatorname{Re} \zeta^{-1}$ and $|\beta| \leqslant 12$. If $\left|\sin \theta / c \lambda_{f}\right|>1$, then $|\zeta|<1$ so $\left|\zeta^{-1}\right|>1$ and $\left|\kappa_{2}\right|$ $\leqslant 1$. We can conclude that $\kappa_{2} \neq \zeta^{-1}$ and that the combined method is stable for the Cauchy problem if $M=1$.

Case II, $M$ Even. If $M$ is even, then $\zeta^{M}=\kappa_{2}$ at $\theta=\pi$ since $\zeta=-1$ and $\kappa_{2}=1$ at $\theta=\pi$. The determinant condition is violated and the combined method unstable for the Cauchy problem for any even $M$.

Case III, $M \geqslant 3$ and Odd. Consider $\theta$ on the interval $\theta_{2} \leqslant \theta \leqslant \theta_{3}$, where $\theta_{2}$ and $\theta_{3}$ are defined as in Lemma 2.1. $\kappa_{2}(\theta)$ is a continuous function of $\theta$ and $\left|\kappa_{2}\right|=1$ on this interval. From Lemma 2.1 we have $\arg \left(\kappa_{2}\left(\theta_{2}\right)\right)=1.797 \ldots$ and $\arg \left(\kappa_{2}\left(\theta_{3}\right)\right)=$ $\pi / 2$ so $\arg \left(\kappa_{2}\left(\theta_{2}\right)\right)>\arg \left(\kappa_{2}\left(\theta_{3}\right)\right)$. It is easily seen [7] that

$$
\zeta=i \sin \theta / c \lambda_{f}+\operatorname{sign}(\cos \theta)\left(1-\sin ^{2} \theta / c^{2} \lambda_{f}^{2}\right)^{1 / 2} .
$$

When $\theta_{2} \leqslant \theta \leqslant \theta_{3}$, then $|\zeta|=1$ and $\operatorname{Im} \zeta=\sin \theta / c \lambda_{f}$ satisfies

$-\frac{1.372 \ldots}{M}=-\frac{\sqrt{36+96 \sqrt{6}}}{12 M}=\frac{\sin \theta_{2}}{c \lambda_{f}} \leqslant \frac{\sin \theta}{c \lambda_{f}} \leqslant \frac{\sin \theta_{3}}{c \lambda_{f}}=-\frac{4}{3 M}=-\frac{1.333 \ldots}{M}$.

$\zeta$ and $\zeta^{-M}$ are also continuous functions on this interval. $\arg (\zeta)=\sin ^{-1}\left(\sin \theta / c \lambda_{f}\right)$ and $\arg \left(\zeta^{-M}\right)=-M \arg (\zeta)$ so $\arg \left(\zeta^{-M}\left(\theta_{2}\right)\right)=-M \sin ^{-1}[(-\sqrt{36+96 \sqrt{6}}) / 12 M]$ and $\arg \left(\zeta^{-M}\left(\theta_{3}\right)\right)=-M \sin ^{-1}(-4 M / 3)$. We consider values of $\sin ^{-1}(\theta)$ on $[0,2 \pi)$. It is clear that $\arg \left(\zeta^{-M}\left(\theta_{2}\right)\right)<\arg \left(\zeta^{-M}\left(\theta_{3}\right)\right)$ and easily seen that $\arg \left(\kappa_{2}\left(\theta_{2}\right)\right)>\arg \left(\zeta^{-M}\left(\theta_{2}\right)\right)$ for all $M \geqslant 3$. Thus, we have two continuous functions, $\kappa_{2}(\theta)$ and $\zeta^{-M}(\theta)$, whose ranges coincide for some interval $\left[\theta_{2}, \theta_{0}\right]$, where $\theta_{2}<\theta_{0} \leqslant \theta_{3}$, so they must take on the same value for some $\theta \in\left[\theta_{2}, \theta_{0}\right]$; and the determinant condition is violated there. By a similar argument we can see that there is another value of $\theta$ between $\theta_{4}$ and $\theta_{5}$ where the determinant condition is violated. Therefore, the combined method is unstable for the Cauchy problem for all odd $M \geqslant 3$. 
The stability of the right quarter-plane problem now follows easily for $M=1$ since $(2.2 b)$ is stable with (2.2a). This results from the fact that we can represent the $v_{\nu}$ in terms of $\zeta$ and the determinantal condition to be verified is just that for (2.2a) with (2.2b) which has already been verified [4]. We have

THEOREM 2.1. The approximation (2.1)-(2.3) is stable for $M=1$ and unstable for all $M \geqslant 2$.

Before commenting on this result we will first present a modified version of this method.

It is of interest to consider handling the right boundary with the $r_{\nu}$ mesh extending from $x=1-2 h_{c}$ to $x=1$ (over two $h_{c}$ intervals as we have done with the left boundary). This is natural to consider for vector equations where there are both inflow and outflow quantities on both boundaries, and for equations with coefficients which are functions of $t$ so that the artificial internal boundary at $x=1-h_{c}$ may be at times an inflow and at times an outflow boundary. We can accomplish this by redefining the grid function $r_{\nu}(t)$ for $\nu=0,1, \ldots, 2 M$ as $r_{\nu}(t)=r\left(1-2 h_{c}+\nu h_{f}, t\right)$ and using the equations

$$
r_{2 M}(t)=g(t), \quad r_{M}(t)=v_{N-1}(t), \quad r_{0}(t)=v_{n-2}(t)
$$

instead of those involving the $r_{\nu}$ of (2.1c).

Let us consider the stability of this method. The associated right quarter-plane problem is the same as before and, therefore, stable if and only if $M=1$. We now consider the associated left quarter-plane problem. Since (2.3a) is stable with (2.3b) as previously remarked we need only consider the stability of (2.1a) coupled with (2.3a) by the conditions $(2.1 \mathrm{e})$ for the related Cauchy problem. If we fold the $x$-axis at $x=1$ and renumber the $v_{\nu}$ and $r_{\nu}$ we again obtain the conditions (2.4) which we have already examined. We have

THEOREM 2.2. The approximation (2.1)-(2.3) with the $r_{\nu}$ approximation extended over $\left[1-2 h_{c}, 1\right]$ and the $r_{\nu}(t)$ equations of $(2.1 \mathrm{c})$ replaced by those of $(2.1 \mathrm{e})$ is stable for $M=1$ and unstable for all $M \geqslant 2$.

The methods found to be unstable in Theorems 2.1 and 2.2 have only violated the determinant condition for values of $z$ which lie on the unit circle, i.e., they satisfy the Godunov-Ryabenkii condition [7]. It is easily seen that the roots $\kappa_{2}(z)$ and $\zeta(z)$ are simple roots of the characteristic equations for those $z$ which violate the determinant condition. Such instabilities have been discussed by Kreiss [8]. Approximations of this type for problems on bounded $x$-intervals have solutions which grow like $N^{\alpha t}$, $\alpha>0$. Further, the extension of any estimates obtainable for problems with constant coefficients to problems with variable coefficients is, in general, impossible.

Computational experiments with $M>1$ for the model problem (1.1)-(1.3) have indicated that these methods can be used successfully for limited times to approximate smooth solutions. However, experiments with the equation $u_{t}-u_{x}-u_{y}=0,0 \leqslant$ $x \leqslant 1,0 \leqslant y \leqslant 1$, have shown disastrous growth when $M$ is even while behaving reasonably for limited times with $M$ odd.

Theorems 2.1 and 2.2 are disappointing. If we couple leapfrog with the centered 
$\mathcal{O}\left(h_{c}^{4}+k^{2}\right)$ interior approximation, we obviously have no opportunity to refine the mesh to achieve uniform accuracy. Computational results with $M=1$ are given in Section 3. They illustrate the fact that we really need $M>1$ to achieve overall $O\left(h_{c}^{4}+k^{2}\right)$ accuracy when compared with results obtained in [11]. However, there are situations where these techniques with $M=1$ can be useful. If the boundary data is rather inaccurate, then nothing could be gained by a refinement, $M>1$. If this is the case and the boundary is sufficiently removed from an interior portion of the domain where the approximation is desired, then these techniques with $M=1$ can be useful. Of course, the area of integration must be so large that the boundary errors will not propagate into the region of interest during the duration of the computation.

We next consider replacing the approximations (2.2) and (2.3) by the dissipative Lax-Wendroff method. We replace (2.2a) by

$$
\begin{array}{r}
l_{\nu}(t+k)=l_{\nu}(t)-k c D_{0}\left(h_{f}\right) l_{\nu}(t)+1 / 2 k^{2} c^{2} D_{+} D_{-} l_{\nu}(t) \\
\text { for } \nu=1,2, \ldots, 2 M-1,
\end{array}
$$

where $D_{+} D_{-} l_{\nu}(t)=\left(l_{\nu+1}(t)-2 l_{\nu}(t)+l_{\nu-1}(t)\right) h_{f}^{-2}$. We replace $(2.2 \mathrm{~b})$ by

$$
l_{0}(t+k)=l_{0}(t)-k c D_{+} l_{0}(t)
$$

where

$$
D_{+} l_{0}(t)=\left(l_{1}(t)-l_{0}(t)\right) h_{f}^{-1} .
$$

Similarly, we replace $(2.3 \mathrm{a})$ by

$$
r_{\nu}(t+k)=r_{\nu}(t)-k c D_{0}\left(h_{f}\right) r_{\nu}(t)+1 / 2 k^{2} c^{2} D_{+} D_{-} r_{\nu}(t)
$$

and $(2.3 b)$ by

$$
\text { for } \nu=1,2, \ldots, M-1 \text {, }
$$

$$
r_{0}(t+k)=r_{0}(t)-k c D_{+} r_{0}(t)
$$

The approximations (2.7a) and (2.8a) have local truncation error $O\left(h_{f}^{2}+k^{2}\right)$, and the boundary approximations $(2.7 \mathrm{~b})$ and $(2.8 \mathrm{~b})$ have local truncation error $\mathrm{O}\left(h_{f}+k\right)$. The approximations (2.7) and (2.8) have been shown to be stable for the related quarterplane problems in [7], and the convergence results of Gustafsson [6] apply in this case as before to tell us that the overall convergence will not be adversely affected if the method is stable. When we apply the same techniques to this method, we again obtain the determinantal conditions (2.4). In this case $\zeta$ is the root of

$$
(z-1) \zeta-\frac{\lambda_{f} c}{2}\left(\zeta^{2}-1\right)-\frac{\lambda_{f}^{2} c^{2}}{2}(\zeta-1)^{2}=0
$$

such that $|\zeta| \leqslant 1$ for $|z| \geqslant 1$. It was shown in [7] that this condition uniquely defines $\zeta$, that $|\zeta|<1$ if $|z| \geqslant 1$ and $c>0$, and $|\zeta|<1$ if $|z| \geqslant 1, z \neq 1$ and $c<0$. If $c<0$ and $z=1$, then $\zeta=1$. Therefore, the determinantal conditions (2.4) are satisfied for all $M$ since $\left|\kappa_{i}\right| \leqslant 1, i=1,2$, and $\left|\zeta^{-M}\right|>1$. If we consider the refinement over two intervals on the right-hand end of the interval, stability is again equivalent to the conditions (2.4) which we have already verified. We have established

THEOREM 2.3. The method given by (2.1), (2.7) and (2.8) with the matching 
conditions (2.1c) is stable for all $M$. The analogous method resulting from the extension of $r_{\nu}$ over $\left[1-2 h_{c}, 1\right]$ and the replacement of the $r_{\nu}$ equations of $(2.1 \mathrm{c})$ by those of (2.1e) is also stable for all $M$.

We present results for this method in Section 3.

It is now easy to see how these results generalize to systems of equations and that the form of the results is independent of the approximations used to a great extent.

Consider the strictly hyperbolic system

$$
u_{t}=A u_{x}, \quad a \leqslant x \leqslant b, t \geqslant 0,
$$

where $u \in R^{s}$ and $A$ is a constant $s \times s$ matrix of the form

$$
A=\left(\begin{array}{cc}
A^{\mathrm{I}} & 0 \\
0 & A^{\mathrm{II}}
\end{array}\right), \quad A^{\mathrm{I}}<0, A^{\mathrm{II}}>0 .
$$

To simplify matters we assume that $A$ has already been transformed to diagonal form.

Let us prescribe initial conditions $u(x, 0)=f(x)$ and boundary conditions

and

$$
u^{\mathrm{I}}=S_{a} u^{\mathrm{II}}+g_{a}^{\mathrm{I}}(t) \quad \text { at } x=a
$$

$$
u^{\mathrm{II}}=S_{b} u^{\mathrm{I}}+g_{b}^{\mathrm{II}}(t) \quad \text { at } x=b
$$

where $u=\left(u^{\mathrm{I}}, u^{\mathrm{II}}\right)^{\prime}$ is partitioned with $A$ and $S_{a}$ and $S_{b}$ are constant rectangular matrices, see [7] for this notation. We assume that this problem is well-posed with the prescribed boundary conditions.

We introduce vector grid functions $l_{\nu}(t), v_{\nu}(t)$, and $r_{\nu}(t)$ as before and denote approximate methods for these three grid functions by $A_{1}, A_{2}$ and $A_{3}$, respectively. We assume that $A_{1}$ and $A_{3}$, with their boundary approximations, are stable for the related quarter-plane and Cauchy problems and that $A_{2}$ is stable for the related Cauchy problem for the given $\lambda_{c}$ and $\lambda_{f}$ defined as before.

In this situation it is more natural to consider the second method of linking the net functions together. That is, we link the grid functions at both ends of the interval requiring equality at some number of points on the $v_{\nu}(t)$ grid. Under the assumption that the methods $A_{1}$ and $A_{3}$ are stable for the related quarter-plane problems, we need only look at the stability of the related Cauchy problems for the combined $A_{1}-A_{2}$ and $A_{2}-A_{3}$ methods; and we can do this separately. We only consider one case; the other is similar. If we look at the related folded problem for $A_{1}-A_{2}$ for $a \leqslant x<\infty$, it is an approximation for the modified equation

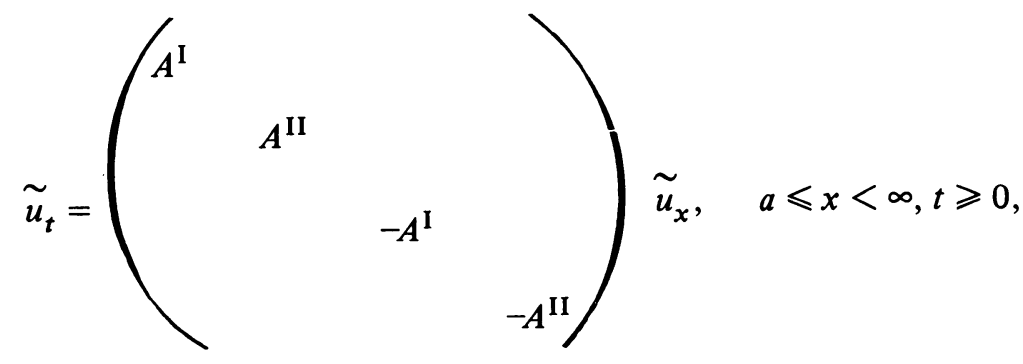


with

$$
\widetilde{u}=\left(\tilde{u}^{\mathrm{I}}, \widetilde{u}^{\mathrm{II}}, \tilde{u}^{\mathrm{III}}, \widetilde{u}^{\mathrm{IV}}\right)
$$

and boundary conditions

$$
\tilde{u}^{\mathrm{I}}=\tilde{u}^{\mathrm{III}}, \quad \tilde{u}^{\mathrm{IV}}=\tilde{u}^{\mathrm{II}} \quad \text { at } x=a .
$$

We then write out the appropriate modified approximation using method $A_{2}$ for the vector $\left(\tilde{u}^{\mathrm{I}}, \widetilde{u}^{\mathrm{II}}\right)^{\prime}$ and $A_{1}$ for the vector $\left(\tilde{u}^{\mathrm{III}}, \widetilde{u}^{\mathrm{IV}}\right)$. The characteristic equations $K_{1}(\zeta, z)$ and $K_{2}(\kappa, z)$ related to the approximations $A_{1}$ and $A_{2}$, respectively, are polynomials in $\kappa$ and $\zeta$, say, with coefficients which are polynomials in $z$. Since $A_{1}$ and $A_{2}$ are stable for the Cauchy problem the roots of $K_{1}$ and $K_{2}$ split into two groups as before, $M_{1, K_{i}}$ and $M_{2, K_{i}}$, with the property that $\left|\kappa_{i}\right| \leqslant 1$ and $\left|\zeta_{i}\right| \leqslant 1$ for $|z| \geqslant 1$ if $\zeta_{i} \in M_{1, K_{1}}$ and $\kappa_{i} \in M_{1, K_{2}}$, and $\left|\kappa_{i}\right| \geqslant 1$ and $\left|\zeta_{i}\right| \geqslant 1$ for $|z| \geqslant 1$ if $\zeta_{i} \in M_{2, K_{1}}$ and $\kappa_{i} \in M_{2, K_{2}}$. This is shown in [7] and simply follows from stability for the related Cauchy problems. Let $M_{1, K_{1}}$ contain $m_{1}$ roots and $M_{1, K_{2}}$ contain $m_{2}$ roots.

Then the $m_{1}+m_{2}$ conditions

$$
v_{\nu}(t)=l_{M \nu}(t), \quad \nu=0,1, \ldots,\left(m_{1}+m_{2}-1\right),
$$

for the original problem uniquely determine the solutions in $l_{2}$. We obtain the determinantal condition $\operatorname{det} C \neq 0$ as before. In this case $C$ is equivalent to a block Vandermonde or block confluent Vandermonde matrix. In fact, if $D$ is the matrix we would obtain using the approximations $A_{1}$ and $A_{2}$ for $u \in R^{1}$ and $D=\left(d_{i j}\right)$, then we can represent $C$ as

$$
C=\left(d_{i j} I_{s}\right)
$$

and $\operatorname{det} C=0$ if and only if $\kappa_{i}=\zeta_{j}^{-M}$ for $\kappa_{i} \in M_{1, K_{2}}$ and $\zeta_{j} \in M_{1, K_{1}}$. Thus, we see that analogs of Theorems 2.1, 2.2 and 2.3 hold in these more general circumstances. In particular, the matching theorem of Ciment [3] holds if only one of the stable matched schemes is dissipative. Details on the derivation of the form of $C$ are given in [3]. We summarize in

Theorem 2.4. The method given by the combination of $A_{1}, A_{2}$, and $A_{3}$ through conditions of the form (2.10) is stable if $\zeta_{j}^{-M} \neq \kappa_{i}$ for $|z| \geqslant 1$, where the $\zeta_{j}$ 's are roots of the characteristic equations corresponding to the boundary methods $A_{1}$ and $A_{3}$ such that $\left|\zeta_{j}\right| \leqslant 1$ and the $\kappa_{i}$ 's are the roots of the characteristic equation corresponding to the interior approximation $A_{2}$ such that $\left|\kappa_{i}\right| \leqslant 1$, for $|z| \geqslant 1$. In particular, if $A_{2}$ is dissipative, or both $A_{1}$ and $A_{3}$ are dissipative, and the root condition $\kappa_{i} \neq \zeta_{j}^{-M}$ holds for $z= \pm 1$, then the combined method is stable.

Proof. To complete the proof we only need to remark that the roots of the characteristic equation for a dissipative approximation [7] satisfy $|\kappa|<1$ for $|z| \geqslant 1$ and $z \neq \pm 1$.

The paper of Gustafsson et al. [7] presents several stable boundary approximations which can be used for $A_{1}$ and $A_{3}$.

Theorem 2.4 shows that dissipative modifications of the leapfrog method could 
be used with the $O\left(h_{c}^{4}+k^{2}\right)$ centered approximation treated in Theorems 2.1 and 2.2 to yield a stable method with $M \geqslant 3$ and odd.

So far our discussion has always assumed that we use the same time step, $k>0$, for both the interior and boundary approximations. The stability restriction on $\lambda_{c}$ places a restriction on $M$ since $\lambda_{f}<\lambda_{c}$ and $\lambda_{c}$ is an increasing function of $M$. For the $O\left(h_{c}^{4}+k^{2}\right)$ and $O\left(h_{f}^{2}+k^{2}\right)$ methods this usually does not cause any real problem. It is natural to choose $h_{c}$ and $k$ so that the truncation errors arising from the spacial and temporal discretizations are of roughly the same size. This leads us to the condition

$$
h_{c}^{4} \approx k^{2} \quad \text { or } \quad h_{c}^{2} \approx k
$$

It is reasonable to choose $M$ so that

$$
h_{f}^{2} \approx h_{c}^{4} \text { or } h_{c}^{2} \approx h_{f}
$$

so that the spatial truncation error is of roughly the same size for the interior and boundary approximations. This leads us to

$$
h_{f} \approx k \text { or } \lambda_{f}=k / h_{f} \approx 1,
$$

which indicates that the usual stability restrictions for explicit methods will not create a problem. This also agrees with the condition we obtain if we ask that the spatial and temporal truncation errors be of the same size in the boundary approximation, i.e., $h_{f}^{2} \approx k^{2}$. The computational results in Section 3 bear this out.

If a situation arises where the previous estimates are not valid, due to the behavior of the solution, a smaller time step can be used on the refined grid by interpolating in time on the $v_{\nu}$ net where intermediate values are needed after first computing the new values on the $v_{\nu}$ net. This is always possible with an explicit method. The previous analysis does not hold in this case but we have performed several computations in this manner which indicate the success of this procedure. Some calculations of this type are presented in Section 3.

3. Computational Results. Our first set of computations are approximations to (1.1), (1.2) and (1.3) with $c=1, a=0, b=1, f(x)=\sin 4 \pi x$ and $g(t)=f(-t)$ which has the solution $u(x, t)=f(x-t)$. This could be stated as a periodic boundary problem but we treat it as an initial boundary-value problem. This is useful since it allows direct comparisons with periodic computations as done in [11]. It was somewhat more convenient to discuss our theoretical results with $c<0$ but we have chosen to use $c=1>0$ here so that the computations will be immediately comparable with those of [11]. The theoretical results are, of course, unchanged and the difference approximations are just the reflections of those already introduced.

We define the error in the $\nu$ th grid point to be $e_{\nu}(t)=u\left(x_{\nu}, t\right)-v_{\nu}(t)$ and compute error norms over the $v_{\nu}(t)$ grid. We use the previously defined $l_{2}\left(h_{c}\right)$ norm and the $l_{\infty}$ norm defined as $\left\|e_{\nu}\right\|_{l_{\infty}}=\max \left|e_{\nu}\right|$.

In Table 3.1 we give the results of the method analogous to that defined by Eqs. (2.1)-(2.3). We include results obtained using smaller time steps on the refined grid and use $L=k_{c} / k_{f}$ to denote this ratio in the table. We append the letters $q$ or $l$ to 
TABLE 3.1

\begin{tabular}{|c|c|c|c|c|c|c|c|}
\hline M & $\mathrm{L}$ & $\left\|v_{v}\right\|_{\ell_{2}}$ & $\left\|e_{v}\right\|_{\ell_{2}}$ & $\left\|e_{\nu}\right\|_{\ell}$ & $\left\|v_{v}\right\|_{\ell_{2}}$ & $\left\|e_{v}\right\|_{\varepsilon_{2}}$ & $\left\|e_{v}\right\|_{\ell}$ \\
\hline & & \multicolumn{3}{|c|}{$t=0.5$} & \multicolumn{3}{|c|}{$t=1.0$} \\
\hline 1 & 1 & $6.54-1$ & $1.14-1$ & $2.23-1$ & $5.71-1$ & $1.85-1$ & $4.16-1$ \\
\hline 2 & 1 & $7.02-1$ & $1.76-2$ & $3.63-2$ & $6.99-1$ & $2.69-2$ & $4.97-2$ \\
\hline 3 & 1 & $7.04-1$ & $6.16-3$ & $1.19-2$ & $7.00-1$ & $9.28-3$ & $1.78-2$ \\
\hline 4 & 1 & $7.07-1$ & $3.02-3$ & $5.55-3$ & $7.07-1$ & $4 \cdot 38-3$ & $9.26-3$ \\
\hline 4 & $2, q$ & $7.06-1$ & $5.99-3$ & $1.14-2$ & $7.05-1$ & $9.48-3$ & $1.95-2$ \\
\hline 4 & $2, l$ & $7.06-1$ & $5.99-3$ & $1.14-2$ & $7.05-1$ & $9.48-3$ & $1.95-2$ \\
\hline 5 & $2, q$ & $7.06-1$ & $4.13-3$ & $8.00-3$ & $7.04-1$ & $5.98-3$ & $1.20-2$ \\
\hline 5 & $2, l$ & $7.06-1$ & $4 \cdot 42-3$ & $8.59-3$ & $7.04-1$ & $6.18-3$ & $1.38-2$ \\
\hline
\end{tabular}

\begin{tabular}{lllllllll} 
& \multicolumn{9}{c}{$t=2.0$} & & \multicolumn{3}{c}{$t=4.0$} \\
\cline { 3 - 5 } 1 & 1 & $5.35-1$ & $2.21-1$ & $4.07-1$ & & $5.37-1$ & $2.03-1$ & $3.76-1$ \\
2 & 1 & $7.02-1$ & $2.39-2$ & $5.81-2$ & & $6.94-1$ & $7.45-2$ & $1.60-1$ \\
3 & 1 & $6.98-1$ & $9.67-3$ & $1.76-2$ & & $6.99-1$ & $9.63-3$ & $1.75-2$ \\
4 & 1 & $7.07-1$ & $4.43-3$ & $9.65-3$ & & $7.07-1$ & $4.76-3$ & $9.99-3$ \\
4 & $2, q$ & $7.06-1$ & $8.85-3$ & $2.20-2$ & & $7.06-1$ & $8.95-3$ & $2.21-2$ \\
4 & $2, l$ & $7.06-1$ & $8.85-3$ & $2.20-2$ & & $7.06-1$ & $8.95-3$ & $2.21-2$ \\
5 & $2, q$ & $7.03-1$ & $5.51-3$ & $1.05-2$ & & $7.03-1$ & $5.54-3$ & $1.05-2$ \\
5 & $2, l$ & $7.04-1$ & $4.83-3$ & $9.74-3$ & $7.04-1$ & $4.90-3$ & $1.01-2$
\end{tabular}

the numbers in the $L$-column to indicate whether quadratic or linear interpolation was used. We have used $\lambda_{c}=1 / 4$ with $N=20$ for these calculations. We have used the solution at $t=k$ for $w$ in (2.1d). We use the notation $a-b$ to represent $a \times 10^{-b}$ in our tables. Recall that these methods are not stable according to the Definition 3.3 of [7] for $M>1$.

In Table 3.2 we report the results of the same computation using Lax-Wendroff in the refined regions, i.e., the reflections of Eqs. (2.1), $\left(2.2 \mathrm{a}^{\prime}\right),\left(2.2 \mathrm{~b}^{\prime}\right),\left(2.3 \mathrm{a}^{\prime}\right)$ and $\left(2.3 b^{\prime}\right)$.

These results can be compared with those given in [11]. We include some results obtained in that paper using uncentered $O\left(h_{c}^{3}\right)$ approximations in the neighborhood of the boundaries for purposes of comparison. The problem and all other parameters are the same as those used here. These results are in Table 3.3.

It is clear that we only need $M=3$ and $L=1$ in this case to achieve the same accuracy. Interpolation in time is not necessary to obtain this accuracy. If greater accuracy is required interpolation may become necessary. 
TABLE 3.2

\begin{tabular}{|c|c|c|c|c|c|c|c|}
\hline M & L & $\left\|v_{v}\right\|_{\ell_{2}}$ & $\left\|e_{v}\right\|_{\ell_{2}}$ & $\left\|e_{\nu}\right\|_{\ell}$ & $\left\|v_{v}\right\|_{\ell_{2}}$ & $\left\|\mathrm{e}_{v}\right\|_{\ell_{2}}$ & $\left\|e_{v}\right\|_{\ell_{\infty}}$ \\
\hline & & \multicolumn{3}{|c|}{$t=0.5$} & \multicolumn{3}{|c|}{$t=1.0$} \\
\hline 1 & 1 & $6.52-1$ & $9.97-2$ & $1.97-1$ & $5.75-1$ & $1.60-1$ & $3.44-1$ \\
\hline 2 & 1 & $6.96-1$ & $1.66-2$ & $3.32-2$ & $6.84-1$ & $2.52-2$ & $3.86-2$ \\
\hline 3 & 1 & $7.04-1$ & $5.97-3$ & $1.09-2$ & $7.00-1$ & $9.02-3$ & $1.39-2$ \\
\hline 4 & 1 & $7.07-1$ & $3.02-3$ & $5.55-3$ & $7.07-1$ & $4 \cdot 38-3$ & $9.26-3$ \\
\hline 4 & $2, q$ & $7.04-1$ & $5.51-3$ & $9.64-3$ & $7.01-1$ & $8.44-3$ & $1.39-2$ \\
\hline 4 & $2, \ell$ & $7.04-1$ & $5 \cdot 56-3$ & $9.27-3$ & $7.01-1$ & $8 \cdot 32-3$ & $1.43-2$ \\
\hline 5 & $2, q$ & $7.05-1$ & $4.11-3$ & $6.32-3$ & $7.04-1$ & $6.21-3$ & $1.17-2$ \\
\hline \multirow[t]{2}{*}{5} & $2, l$ & 1.05-1 & $4.18-3$ & $6.67-3$ & $7.04-1$ & $6.14-3$ & $1.21-2$ \\
\hline & & \multicolumn{3}{|c|}{$t=2.0$} & \multicolumn{3}{|c|}{$t=4.0$} \\
\hline 1 & 1 & $5.48-1$ & $1.88-1$ & $3 \cdot 32-1$ & $5.52-1$ & $1.77-1$ & $3.14-1$ \\
\hline 2 & 1 & $6.83-1$ & $2.69-2$ & $3.98-2$ & $6.83-1$ & $2.70-2$ & $4.05-2$ \\
\hline 3 & 1 & 6.99-1 & $9.41-3$ & $1.52-2$ & $6.99-1$ & $9.42-3$ & $1.53-2$ \\
\hline 4 & 1 & $7.07-1$ & $4 \cdot 43-3$ & $9.66-3$ & $7.07-1$ & $4 \cdot 73-3$ & $9 \cdot 91-3$ \\
\hline 4 & $2, q$ & $7.01-1$ & $8.78-3$ & $1.49-2$ & $7.01-1$ & $8.79-3$ & $1.50-2$ \\
\hline 4 & $2, l$ & $7.01-1$ & $8.20-4$ & $1.45-2$ & $7.01-1$ & $8.23-3$ & $1.48-2$ \\
\hline 5 & $2, q$ & $7.03-1$ & $6.33-3$ & $1.16-2$ & $7.03-1$ & $6.34-3$ & $1.17-2$ \\
\hline 5 & $2, l$ & $7.04-1$ & $5.76-3$ & $1.13-2$ & $7.04-1$ & $5.79-3$ & $1.15-2$ \\
\hline
\end{tabular}

TABLE 3.3

\begin{tabular}{|c|c|c|c|c|c|}
\hline$\left\|v_{v}\right\|_{\ell_{2}}$ & $\left\|e_{v}\right\|_{l_{2}}$ & $\left\|e_{\nu}\right\|_{\ell_{\infty}}$ & $\left\|v_{v}\right\|_{\ell_{2}}$ & $\left\|e_{v}\right\|_{\ell_{2}}$ & $\left\|e_{\nu}\right\|_{\ell_{\alpha}}$ \\
\hline & $t=0.5$ & & & $t=1.0$ & \\
\hline $7.12-1$ & $\begin{array}{l}9.69-3 \\
t=2.0\end{array}$ & $2.34-2$ & $7.08-1$ & $\begin{array}{l}1.34-2 \\
t=4.0\end{array}$ & $2.51-2$ \\
\hline $6.96-1$ & $1.30-2$ & $2.04-2$ & $6.96-1$ & $1.25-2$ & $2.28-2$ \\
\hline
\end{tabular}

Acknowledgments. Programs for tests of the root conditions and the experiments for $u_{t}=u_{x}$ in Section 3 were written by John Bolstad and Michael Heath of the Stanford Computer Science Department. 
1. G. BROWNING, H.-O. KREISS \& J. OLIGER, "Mesh refinement," Math. Comp., v. 27, 1973, pp. 29-39. MR 48 \# 12861.

2. M. CIMENT, "Stable difference schemes with uneven mesh spacings," Math. Comp., v. 25 , 1971, pp. 219-227. MR 45 \# 9516.

3. M. CIMENT, "Stable matching of difference schemes," SIAM J. Numer. Anal., v. 9, 1972, pp. 695-701. MR 47 \# 7927.

4. T. ELVIUS \& A. SUNDSTRÖM, "Computationally efficient schemes and boundary conditions for a fine-mesh barotropic model based on the shallow-water equations," Tellus, v. 25, 1973, pp. $132-156$.

5. B. FORNBERG, On High Order Approximations of Hyperbolic Partial Differential Equations by a Fourier Method, Report 39, Dept. of Computer Sciences, Uppsala University, 1972.

6. B. GUSTAFSSON, On the Convergence Rate for Difference Approximations to Mixed Initial Boundary Value Problems, Report 33, Dept. of Computer Sciences, Uppsala University, 1971.

7. B. GUSTAFSSON, H.-O. KREISS \& A. SUNDSTRÖM, "Stability theory of difference approximations for mixed initial boundary-value problems. II," Math. Comp., v. 26, 1972, pp. 649686. MR 49 \# 6634.

8. H.-O. KREISS, "Difference approximations for initial boundary-value problems," Proc. Roy. Soc. London Ser. A, v. 323, 1971, pp. 255-261.

9. H.-O. KREISS \& J. OLIGER, "Comparison of accurate methods for the integration of hyperbolic equations," Tellus, v. 24, 1972, pp. 199-215. MR 47 \# 7926.

10. M. MARDEN, Geometry of Polynomials, 2nd ed., Math. Surveys, no. 3, Amer. Math. Soc., Providence, R. I., 1966. MR 37 \# 1562.

11. J. OLIGER, "Fourth order difference methods for the initial boundary-value problem for hyperbolic equations," Math. Comp., v. 28, 1974, pp. 15-25. MR 50 \#11798. 